\title{
Staphylococcus aureus bloodstream infection in Australian hospitals: findings from a Victorian surveillance system
}

\section{Leon J Worth \\ MBBS, FRACP, PhD Infectious Diseases Physician \\ Tim Spelman MBBS, BSc, GradCert(Stats) Biostatistician \\ Ann LBull \\ Epidemiologist \\ Michael J Richards MBBS, FRACP, MD \\ Director \\ Victorian Healthcare Associated Infection \\ Surveillance System \\ Coordinating Centre, \\ Melbourne, VIC}

leon.worth@mh.org.au

MJA 2014; 200: 282-284 doi: 10.5694/mjal3.10599
西 ealth care-associated (HA) infections are an important measure of quality of care in health care facilities. Staphylococcus aureus bloodstream (SAB) infections contribute to morbidity, mortality, and health care expenditure, and are frequently regarded as preventable. ${ }^{1}$

Standardised surveillance strategies for SAB infections have been effectively implemented internationally. 2,3 In 2008, the Australian Health Ministers' Conference endorsed that all Australian hospitals should establish HA infection surveillance, including reporting of SAB infections through relevant jurisdictions to a national data repository.

The Victorian Healthcare Associated Infection Surveillance System (VICNISS) SAB infection surveillance module was developed to enable collection of relevant laboratory and clinical data by health care workers trained in infectious diseases or infection prevention for community and HA-SAB infection events in the state of Victoria, including methicillin-sensitive and methicillin-resistant isolates.

The Victorian Department of Health mandated participation by Victorian public hospitals at the commencement of the program. VICNISS provided education to participating hospitals and developed a guide to facilitate standardised case review. Private health care facilities participated on a voluntary basis.

In Victoria, reporting of SAB infection events commenced in October 2009. In January 2010, the VICNISS implemented a standardised module that uses definitions developed by the Australian Commission on Safety and Quality in Health Care (ACSQHC) for monitoring in Victorian acute care public hospitals. This report outlines module components and a review of surveillance data captured during the first 3 years.

\section{Abstrac}

Objectives: To determine the burden of disease and trend over time for rates of Staphylococcus aureus bloodstream (SAB) infections in Victorian health care services.

Design and setting: Uniform data on all SAB infection events (methicillinsensitive and methicillin-resistant isolates) were collected from all public and some private hospitals in Victoria using a standardised electronic data collection tool. Data were analysed for the period 1 January 2010 to 31 December 2012.

Main outcome measures: Overall and quarterly aggregate SAB and methicillinresistant S. aureus (MRSA) bloodstream infection rates per 10000 occupied bed-days (OBDs); rates of health care-associated (HA) infections compared with a benchmark of no more than 2/10 000 OBDs.

Results: Data from 119 public and four private hospitals were analysed. The cumulative aggregate SAB infection rate was 1.0/10 000 OBDs $(95 \% \mathrm{Cl}$, 0.9$1.0 / 10000$ OBDs). Overall, $1335 / 3205$ SAB infection events (41.7\%) were health care-associated. Of these, $26.2 \%$ occurred within 48 hours of hospitalisation and were most frequently associated with an indwelling medical device. Quarterly HA-SAB infection rates diminished from 1.4 to $0.7 / 10000$ OBDs $(P<0.001)$. A median of four health care services each quarter exceeded the benchmark of 2.0/10 000 OBDs. HA-MRSA bloodstream infection rates diminished from 0.4 to $0.1 / 10000$ OBDs $(P<0.001)$, with a cumulative aggregate rate of $0.2 / 10000$ OBDs.

Conclusions: Continuous surveillance for SAB infection showed a significant reduction in rates across Victoria during the first 3 years of a coordinated program. Early onset, device-related SAB infections are an important target for prevention strategies.

\section{Methods}

Uniform data were captured for all SAB infection events, including patient demographics, infection details and organism susceptibility.

Each infection was classified as either HA or community-associated using ACSQHC definitions. SAB infection was defined as HA if:

- the patient's first $S$. aureus-positive blood culture was collected $>48$ hours after admission to hospital or $<48$ hours after discharge, or

- the patient's first $S$. aureus-positive blood culture was collected $\leqslant 48$ hours after admission to hospital and key clinical criteria were also met:

> SAB infection was a complication of the presence of an indwelling medical device;

$>$ SAB infection occurred within 30 days of a surgical procedure where the SAB infection was related to the surgical site;
$>\mathrm{SAB}$ infection was diagnosed within 48 hours of a related invasive instrumentation or incision; or

$>\mathrm{SAB}$ infection was associated with neutropenia contributed to by cytotoxic therapy.

$\mathrm{SAB}$ infection was defined as community-associated if the patient's first S. aureus-positive blood culture was collected $\leqslant 48$ hours after admission to hospital and none of the key clinical criteria listed above were met.

The first SAB isolate per patient was counted as one episode. Subsequent positive blood cultures were only recorded as additional episodes if $\geqslant 14$ days had passed without a positive blood culture. Data for total occupied bed-days (OBDs) were obtained from the Victorian Admitted Episodes Dataset and HA-SAB infection rates were reported as number of epiodes per 10000 OBDs.

\section{Data collection and validation}

Participating hospitals submitted data using a web-based tool. Webform 
design precluded lodgement of incomplete or inconsistent data. VICNISS staff were available if participating hospitals required assistance with applying standard definitions.

\section{Statistical analysis}

For this study, data collected during the first 36 months of the program (1 January 2010 - 31 December 2012) were analysed to determine relative proportions of HA and communityassociated events and the proportion of SAB infection events attributed to methicillin-resistant S. aureus (MRSA). To test the hypothesis that a change in aggregate HA-SAB infection and MRSA bloodstream infection rates was not observed over time, both linear and non-linear quadratic trends in quarterly rates were evaluated using generalised estimating equations for Poisson regression with OBDs as the exposure. As no pre-observation data were available, we were unable to calculate a meaningful rate at time zero and thus suppressed the model intercept terms. The quasi-likelihood information criterion 4 and modelled values for deviance and dispersion were used as metrics to assess comparative goodness of fit for linear and quadratic models. Cubic fits were calculated as a sensitivity analysis regarding the validity of presuming an underlying non-linear quadratic relationship. Statistical tests were performed using Stata, version 12 (StataCorp). Quarterly data for the study period were compared with the national threshold target of no more than 2.0/10000 OBDs for HA-SAB infection rates in Australian hospitals in 2011.

\section{Results}

SAB infection data were submitted by 119 public health care facilities spanning 90/90 health care services (100\%), and four private health care facilities. During the period studied, a total of 3205 SAB infection events were captured (2072 male patients, 1132 female patients, sex unknown for one event). The median age of patients with SAB infection was 64 years (range, 0-104 years).

Of all reported infection events, 1335 (41.7\%) were HA, 1803 (56.3\%) were community-associated, and 67 (2.1\%) were unknown or unable to be classified. Of the HA-SAB infection events, 350 (26.2\%) occurred $\leqslant 48$ hours after hospital admission and 985 (73.8\%) occurred $>48$ hours after admission. MRSA was responsible for 295 HA-SAB infection events (22.1\%) and 175 community-associated infection events (9.7\%).

Quarterly data for HA-SA and HA-MRSA bloodstream infection events are summarised in Box 1 . The median aggregate quarterly infection rates for HA-SA and HA-MRSA, respectively, were 0.95/10 000 OBDs (range, 0.7-1.4/10 000 OBDs) and 0.2/10000 OBDs (range, 0.1$0.4 / 10000$ OBDs). Linear and quadratic regression models were fitted. The quadratic models both showed a significant decreasing trend over time $(P<0.001)$ with better goodness of fit. The quadratic models showed a decrease of 6.2 HA-SAB infection events per cumulative quarterly OBDs with a 0.5 unit change in slope (the quadratic coefficient), and a decrease of 7.8 HA-MRSA bloodstream infection events per cumulative quarterly OBDs with a 0.6 unit change in slope. The overall cumulative aggregate infection rates were 1.0/10 000 OBDs (95\% CI, 0.9-1.0/10 000 OBDs) for HA-SA and $0.2 / 10000$ OBDs (95\% CI, 0.190.24/10 000 OBDs) for HA-MRSA.

1 Quarterly health care-associated Staphylococcus aureus bloodstream (SAB) infection rates* per 10000 occupied bed-days (OBDs), Victorian health care facilities, † Jan 2010 - Dec 2012

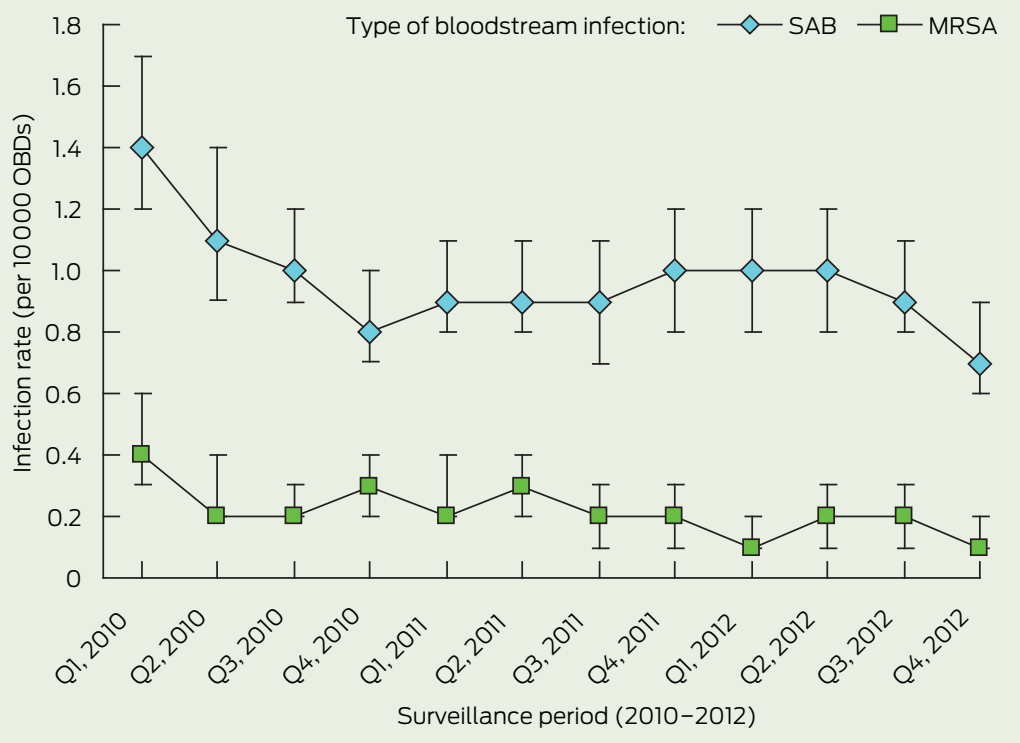

MRSA = methicillin-resistant S. aureus. * Error bars indicate $95 \% \mathrm{Cls} . \dagger 119$ public health care facilities spanning 90/90 health care services (100\%), and four private health care facilities.

2 Key clinical criteria associated with health care-associated Staphylococcus aureus bloodstream ( $S A B$ ) infection occurring $\leqslant 48$ hours after hospital admission $(n=350)$, Victorian health care facilities, * Jan 2010 - Dec 2012

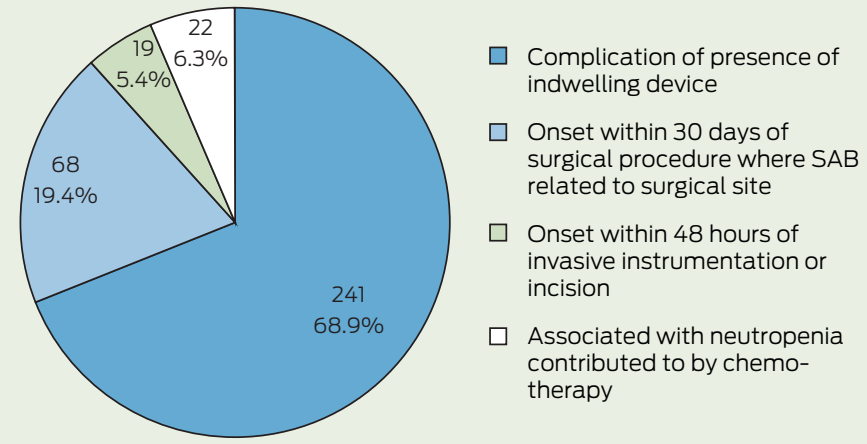

* 119 public health care facilities spanning 90/90 health care services (100\%), and four private health care facilities. 
Quarterly HA-SAB infection rates in excess of 2/10000 OBDs were reported in 54 instances, corresponding to a median of four (range, 2-8) instances each quarter. During the first quarter, eight health care services exceeded the benchmark, while four services breached the threshold target during the final reporting quarter.

Of the 350 HA-SAB infections that were identified $\leqslant 48$ hours after hospital admission, most (68.9\%) were reported as a complication of the presence of an indwelling medical device; $19.4 \%$ occurred within 30 days of a surgical procedure and were related to the surgical site. Smaller numbers of infections were reported in association with invasive instrumentation or in the setting of neutropenia contributed to by cytotoxic therapy. Associated key clinical criteria are summarised in Box 2 .

\section{Discussion}

Our findings reflect successful implementation of continuous statewide SAB infection surveillance using nationally agreed criteria. Further, a significant reduction in Victorian SAB infection rates was shown during the first 3 years. This may be indicative of concurrent infection prevention strategies (eg, improved practices for intravenous catheter insertion and care, ${ }^{5}$ reduced surgical site infections, ${ }^{6}$ an operative hand-hygiene program ${ }^{7}$ ), together with the fact that falling rates of infection are frequently observed after the commencement of formal surveillance programs. ${ }^{8}$

Notably, $26 \%$ of HA-SAB infection events occurred within 48 hours of hospital admission. These early events were frequently (69\%) associated with an indwelling medical device (eg, intravenous catheter, haemodialysis vascular access) present at the time of hospital admission (Box 2). Patients at risk of these events include those with intravenous devices managed by hospital-in-the-home programs, those on haemodialysis, haematology and oncology outpatients with central venous access devices and outpatients receiving parenteral nutrition. Given the burden of illness, surveillance strategies for HA infections must continue to capture early ( $\leqslant 48$ hours after hospital admission), as well as late, events.

MRSA infection has previously been reported to comprise $11 \%-$ $40 \%$ of HA-SAB infection events in Australia, ${ }^{1,9}$ but studies have been limited by the potential for selective sampling. Our statewide data indicated that $22 \%$ of HA-SAB infection events were due to MRSA, which is comparable to recent data from European and Canadian reports. ${ }^{10}$

For SAB infection surveillance, the denominator for a health care service comprises OBDs for acute medical care, acute surgical care, intensive care, hospital-in-the-home, rehabilitation and psychiatric care, in addition to OBDs for rehabilitation or psychiatry centres associated with the nominated acute care hospital. SAB infection events are infrequently seen within psychiatry and rehabilitation facilities. To focus on acute care centres would be an optimal use of infection prevention resources, and a denominator excluding low-risk patients would provide more meaningful data for interhospital comparison.

After the commencement of SAB infection surveillance in Victoria, public reporting was facilitated nationally by the Australian Institute of Health and Welfare via the MyHospitals website. ${ }^{11}$ Based on the National Healthcare Agreement, 12 a threshold target rate for SAB infection (no more than 2.0/10000 patient days) in acute care public hospitals was applied in 2012. ${ }^{13}$ Retrospective application of this target to our data spanning a 36-month period showed that eight health care services were above this target at the onset of the surveillance period, with four identified during the last quarter studied. If hospital-level data are to be compared with a national benchmark, it is vital that jurisdictions implement uniform methods for surveillance ${ }^{14}$ and validation. ${ }^{15}$ Our experience shows that, to ensure uniformity of surveillance methods and for education and discussion of complex events, health care facilities require direct and frequent liaison with a coordinating centre. Consideration of lower threshold targets may be necessary if sustainable improvement, such as that seen in Victoria, is achieved. ${ }^{15}$
Acknowledgements: We acknowledge Victorian infection control consultants for collection of data and feedback regarding module development, and thank Mohammadreza Mohebbi for assistance with the analysis.

\section{Competing interests: No relevant disclosures.}

Received 5 May 2013, accepted 10 Oct 2013.

1 Turnidge JD, Kotsanas D, Munckhof W, et al. Staphylococcus aureus bacteraemia: a major cause of mortality in Australia and New Zealand. Med J Aust 2009; 191: 368-373.

2 Ellington MJ, Hope R, Livermore DM, et al. Decline of EMRSA-16 amongst methicillinresistant Staphylococcus aureus causing bacteraemias in the UK between 2001 and 2007. J Antimicrob Chemother 2010; 65: 446448.

3 Faria NA, Oliveira DC, Westh $\mathrm{H}$, et al. Epidemiology of emerging methicillin-resistant Staphylococcus aureus (MRSA) in Denmark: a nationwide study in a country with low prevalence of MRSA infection. J Clin Microbiol 2005; 43: 1836-1842.

4 Cui J. QIC program and model selection in GEE analysis. The Stata Journal 2007; 7: 209220. http://www.stata-journal.com/sjpdf. html?articlenum=st0126 (accessed Oct 2013).

5 Lee MK, Mossop PJ, Vrazas II. Central venous catheter placement by an interventional radiology unit: an Australian experience. Australas Radiol 2007; 51: 35-41.

6 Bull A, Wilson J, Worth LJ, et al. A bundle of care to reduce colorectal surgical infections: an Australian experience. J Hosp Infect 2011; 78: 297-301.

7 Grayson ML, Jarvie LJ, Martin R, et al. Significant reductions in methicillin-resistant Staphylococcus aureus bacteraemia and clinical isolates associated with a multisite, hand hygiene culture-change program and subsequent successful statewide roll-out. Med J Aust 2008; 188: 633-640.

8 Brandt C, Sohr D, Behnke M, et al. Reduction of surgical site infection rates associated with active surveillance. Infect Control Hosp Epidemiol 2006; 27: 1347-1351.

9 Cordova SP, Heath CH, McGechie DB, et al. Methicillin-resistant Staphylococcus aureus bacteraemia in Western Australian teaching hospitals, 1997-1999: risk factors, outcomes and implications for management. J Hosp Infect 2004; 56: 22-28.

10 Adam HJ, DeCorby M, Rennie R, et al. Prevalence of antimicrobial resistant pathogens from blood cultures from Canadian hospitals: results of the CANWARD 2007-2009 study. Diagn Microbiol Infect Dis 2011; 69: 307-313.

11 National Health Performance Authority. MyHospitals [website]. http://www. myhospitals.gov.au (accessed Jan 2012).

12 Council of Australian Governments. National Healthcare Agreement 2012. http://www. federalfinancialrelations.gov.au/content/npa/ healthcare/national-agreement.pdf (accessed Oct 2013).

13 Kirby T. Australia publishes hospital staphylococcus bacteraemia rates. Lancet Infect Dis 2012; 12: 105-106.

14 Worth LJ, Thursky KA, Slavin MA. Public disclosure of health care-associated infections in Australia: quality improvement or parody [letter]? Med J Aust 2012; 197: 29.

15 Worth LJ, Bull AL, Richards MJ. Public reporting of health care-associated infection data in Australia: time to refine. Med J Aust 2013; 198: 252-253. 